\title{
On chiral-odd Generalized Parton Distributions
}

\section{El Beiyad}

CPHT, École Polytechnique, CNRS, 91128 Palaiseau Cedex, France \&

LPT, Université Paris-Sud, CNRS, 91405 Orsay, France

E-mail: Mounir.Elbeiyadecpht.polytechnique.fr

\section{B. Pire}

CPHT, École Polytechnique, CNRS, 91128 Palaiseau Cedex, France

E-mail: piredcpht.polytechnique.fr

\section{Segond}

Institut für Theoretische Physik, Universität Leipzig, D-04009 Leipzig, Germany

E-mail: Mathieu.Segondeitp.uni-leipzig.de

\section{Szymanowski}

Soltan Institute for Nuclear Studies, PL-00-681 Warsaw, Poland

E-mail: Lech.Szymanowski@fuw.edu.pl

\section{S. Wallon*}

LPT, Université Paris-Sud, CNRS, 91405 Orsay, France \& UPMC Univ. Paris 06, faculté de physique, 4 place Jussieu, 75252 Paris Cedex 05, France

E-mail: walloneth.u-psud.fr

\begin{abstract}
While the chiral-even content of the nucleon is better and better studied, the chiral-odd transversity generalized parton distributions (GPDs) remain almost unknown. These GPDs can be accessed experimentally through the exclusive photoproduction process $\gamma+N \rightarrow \pi+\rho+N^{\prime}$, in the kinematics where the meson pair has a large invariant mass and the final nucleon has a small transverse momentum, provided the vector meson is produced in a transversally polarized state. We calculate perturbatively the scattering amplitude at leading order in $\alpha_{s}$. We build a simple model for the dominant transversity GPD $H_{T}(x, \xi, t)$ based on the concept of double distribution. We estimate the unpolarized differential cross section for this process in the kinematics of the Jlab and COMPASS experiments. Counting rates show that the experiment looks feasible with the real and quasi real photon beam characteristics expected at JLab@12 GeV, and with the quasi real photon beam in the COMPASS experiment.
\end{abstract}

35th International Conference of High Energy Physics

July 22-28, 2010

Paris, France

*Speaker. 


\section{Chiral-odd GPDs and factorization}

Among the leading twist hadronic observables, transversity quark distributions in the nucleon remain the most unknown ones. Indeed their chiral-odd character enforces their decoupling in most hard amplitudes at the leading twist level. After the pioneering studies of Ref. [1], much work [2] has been devoted to the study of many channels but experimental difficulties have challenged the most promising ones. Besides, tremendous progress has been recently witnessed on the QCD description of hard exclusive processes, in terms of generalized parton distributions (GPDs) describing the 3-dimensional content of hadrons. Access to the chiral-odd transversity GPDs [3], noted $H_{T}, E_{T}, \tilde{H}_{T}, \tilde{E}_{T}$, has however turned out to be even more challenging [4] than the usual transversity distributions: one photon or one meson electroproduction leading twist amplitudes are insensitive to transversity GPDs. As initiated in Ref. [5], we here study the leading twist contribution to processes where more mesons are present in the final state ${ }^{1}$; the hard scale which allows to probe the short distance structure of the nucleon is $s=M_{\pi \rho}^{2} \sim\left|t^{\prime}\right|$ in the fixed angle regime. The process of Ref. [5] was the high energy photo (or electro) diffractive production of two vector mesons, the hard probe being the virtual "Pomeron" exchange (the hard scale was the virtuality of this pomeron), in analogy with the virtual photon exchange occuring in the deep electroproduction of a meson. We study here [7] a process involving a transversely polarized $\rho$ meson in a 3-body final state:

$$
\gamma+N \rightarrow \pi+\rho_{T}+N^{\prime} .
$$

It is a priori sensitive to chiral-odd GPDs due to the chiral-odd character of the leading twist distribution amplitude (DA) of $\rho_{T}$. The estimated rate depends of course much on the magnitude of the chiral-odd GPDs. Not much is known about them, but model calculations have been developed in Refs. [5, 8-10] and a few moments have been computed on the lattice [11]. To factorize the amplitude of this process we use the now classical proof of the factorization of exclusive scattering at fixed angle and large energy [12]. The amplitude for the process $\gamma+\pi \rightarrow \pi+\rho$ is written as the convolution of mesonic DAs and a hard scattering subprocess amplitude $\gamma+(q+\bar{q}) \rightarrow(q+\bar{q})+(q+\bar{q})$ with the meson states replaced by collinear quark-antiquark pairs. This is described in Fig.1a. The absence of any pinch singularities (which is the weak point of the proof for the generic case $A+B \rightarrow C+D$ ) has been proven in Ref. [13] for the case of interest here. We then extract from the factorization procedure of the deeply virtual Compton scattering amplitude near the forward region the right to replace in Fig.1a the lower left meson DA by a $N \rightarrow N^{\prime}$ GPD, and thus get Fig.1b. The needed skewness parameter $\xi$ is written in terms of the meson pair squared invariant mass $M_{\pi \rho}^{2}$ as

$$
\xi=\frac{\tau}{2-\tau} \quad, \quad \tau=\frac{M_{\pi \rho}^{2}}{S_{\gamma N}-M^{2}} .
$$

Indeed the same collinear factorization property bases the validity of the leading twist approximation which either replaces the meson wave function by its DA or the $N \rightarrow N^{\prime}$ transition by nucleon GPDs. A slight difference is that light cone fractions $(z, 1-z)$ leaving the DA are positive, while the corresponding fractions $(x+\xi, \xi-x)$ may be positive or negative in the case of the GPD. A Born order calculation show that this difference does not ruin the factorization property. In order for the factorization of a partonic amplitude to be valid, and the leading twist calculation to be suf-

\footnotetext{
${ }^{1}$ A similar strategy has also been advocated recently in Ref. [6] for chiral-even GPDs.
} 
ficient, one should avoid the dangerous kinematical regions where a small momentum transfer
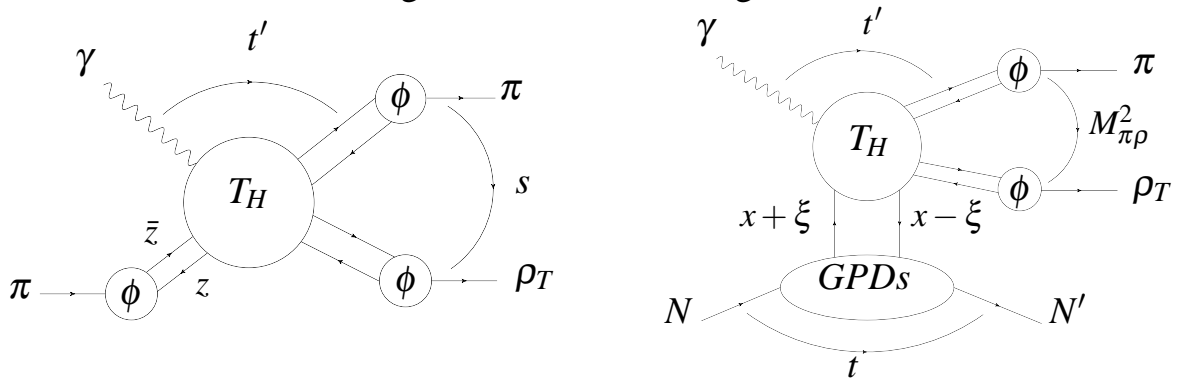

Figure 1: a) (left) Factorization of the amplitude for $\gamma+\pi \rightarrow \pi+\rho$ at large $s$ and fixed angle; b) (right) replacing one DA by a GPD leads to the factorization of the amplitude for $\gamma+N \rightarrow \pi+\rho+N^{\prime}$ at large $M_{\pi \rho}^{2}$.

is exchanged in the upper blob, namely small $t^{\prime}=\left(p_{\pi}-p_{\gamma}\right)^{2}$ or small $u^{\prime}=\left(p_{\rho}-p_{\gamma}\right)^{2}$, and the resonance regions for each of the invariant squared masses $\left(p_{\pi}+p_{N^{\prime}}\right)^{2},\left(p_{\rho}+p_{N^{\prime}}\right)^{2},\left(p_{\pi}+p_{\rho}\right)^{2}$.

\section{The scattering amplitude}
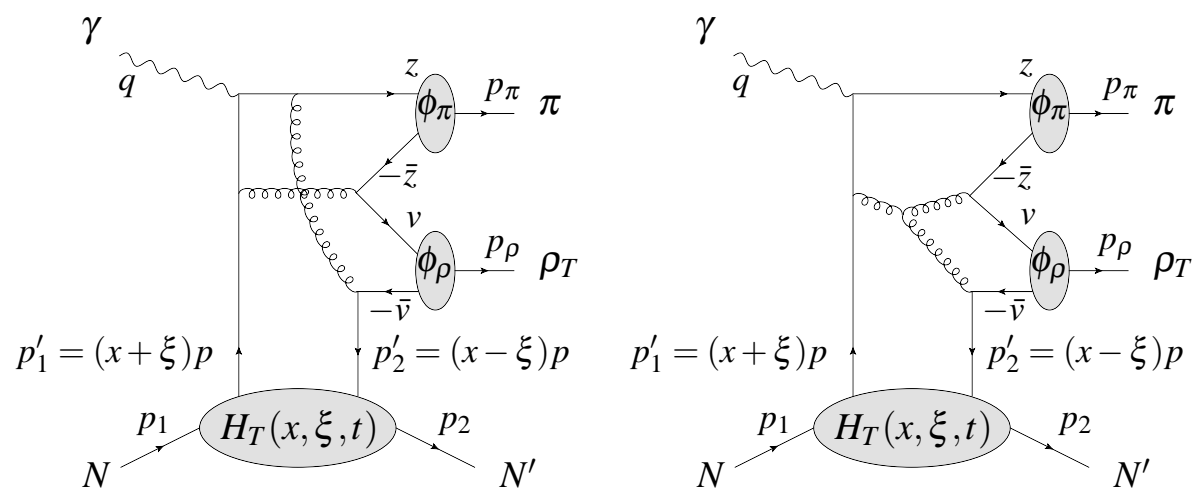

Figure 2: Two representative diagrams without (left) and with (right) three gluon coupling.

The scattering amplitude of the process (1.1) is written in the factorized form :

$$
\mathscr{A}\left(t, M_{\pi \rho}^{2}, p_{T}\right)=\int_{-1}^{1} d x \int_{0}^{1} d v \int_{0}^{1} d z T^{q}(x, v, z) H_{T}^{q}(x, \xi, t) \Phi_{\pi}(z) \Phi_{\perp}(v),
$$

where $T^{q}$ is the hard part of the amplitude and the transversity GPD of a parton $q$ in the nucleon target which dominates at small momentum transfer is defined by [3]

$$
\left\langle N^{\prime}\left(p_{2}\right), \lambda^{\prime}\left|\bar{q}\left(-\frac{y}{2}\right) \sigma^{+j} \gamma^{5} q\left(\frac{y}{2}\right)\right| N\left(p_{1}\right), \lambda\right\rangle=\bar{u}\left(p^{\prime}, \lambda^{\prime}\right) \sigma^{+j} \gamma^{5} u(p, \lambda) \int_{-1}^{1} d x e^{-\frac{i}{2} x\left(p_{1}^{+}+p_{2}^{+}\right) y^{-}} H_{T}^{q},
$$

where $\lambda$ and $\lambda^{\prime}$ are the light-cone helicities of the nucleon $N$ and $N^{\prime}$. The chiral-odd DA for the transversely polarized meson vector $\rho_{T}$, is defined, in leading twist 2 , by the matrix element

$$
\left\langle 0\left|\bar{u}(0) \sigma^{\mu v} u(x)\right| \rho_{T}^{0}\left(p, \varepsilon_{ \pm}\right)\right\rangle=\frac{i}{\sqrt{2}}\left(\varepsilon_{ \pm}^{\mu}(p) p^{v}-\varepsilon_{ \pm}^{v}(p) p^{\mu}\right) f_{\rho}^{\perp} \int_{0}^{1} d u e^{-i u p \cdot x} \phi_{\perp}(u),
$$

where $\varepsilon_{ \pm}^{\mu}\left(p_{\rho}\right)$ is the $\rho$-meson transverse polarization and with $f_{\rho}^{\perp}=160 \mathrm{MeV}$. This process is described by 62 Feynman diagrams (see Fig.2). The scattering amplitude gets both a real and an imaginary parts. Integrations over $v$ and $z$ have been done analytically whereas numerical methods are used for the integration over $x$. 


\section{Results}

Various observables can be calculated with this amplitude. We stress that even the unpolarized differential cross-section $\frac{d \sigma}{d t d u^{\prime} d M_{\pi \rho}^{2}}$ is sensitive to the transversity GPD. To estimate the rates, we modelize the dominant transversity GPD $H_{T}^{q}(x, \xi, t)(q=u, d)$ in terms of double distributions

$$
H_{T}^{q}(x, \xi, t=0)=\int_{\Omega} d \beta d \alpha \delta(\beta+\xi \alpha-x) f_{T}^{q}(\beta, \alpha, t=0),
$$

where $f_{T}^{q}$ is the quark transversity double distribution written as

$$
f_{T}^{q}(\beta, \alpha, t=0)=\Pi(\beta, \alpha) \delta q(\beta) \Theta(\beta)-\Pi(-\beta, \alpha) \delta \bar{q}(-\beta) \Theta(-\beta),
$$

where $\Pi(\beta, \alpha)=\frac{3}{4} \frac{(1-\beta)^{2}-\alpha^{2}}{(1-\beta)^{3}}$ is a profile function and $\delta q, \delta \bar{q}$ are the quark and antiquark transversity parton distribution functions of Ref. [14]. The $t$-dependence of these chiral-odd GPDs - and its Fourier transform in terms of the transverse localization of quarks in the proton [15] - is very interesting but completely unknown. We describe it in a simplistic way using a dipole form factor:

$$
H_{T}^{q}(x, \xi, t)=H_{T}^{q}(x, \xi, t=0) \times \frac{C^{2}}{(t-C)^{2}} \quad\left(C=.71 \mathrm{GeV}^{2}\right) .
$$

In Fig. 3, we show the $M_{\pi \rho}^{2}$ dependence of the differential cross section $d \sigma / d M_{\pi \rho}^{2}$. Let us first
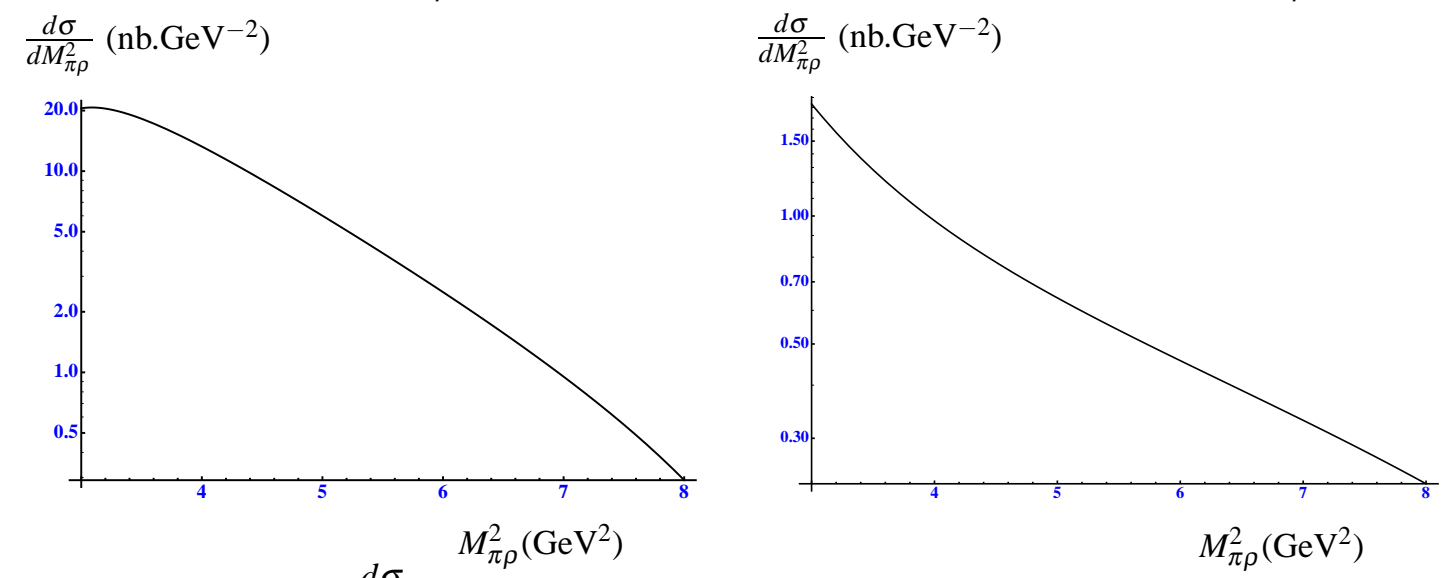

Figure 3: $\frac{d \sigma}{d M_{\pi \rho}^{2}}\left(\mathrm{nb} \mathrm{GeV}^{-2}\right)$ at $S_{\gamma N}=20 \mathrm{GeV}^{2}$ (left) and $S_{\gamma N}=100 \mathrm{GeV}^{2}$ (right).

discuss the specific case of muoproduction with the COMPASS experiment at CERN. Integrating differential cross sections on $t, u^{\prime}$ and $M_{\pi \rho}^{2}$, with justified cuts on $t^{\prime}$ and $u^{\prime}$ and $M_{\pi \rho}^{2}>3 \mathrm{GeV}^{2}$, leads to an estimate of the cross sections for the photoproduction of a $\pi^{+} \rho_{T}^{0}$ pair at high energies such as

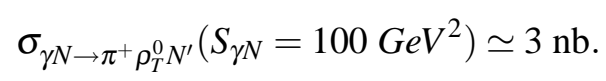

The virtuality $Q^{2}$ of the exchanged photon plays no crucial role in our process, and the virtual photoproduction cross section is almost $Q^{2}$-independent if we choose to select events in a sufficiently narrow $Q^{2}$-window $\left(.02<Q^{2}<1 \mathrm{GeV}^{2}\right.$ ), which is legitimate since the effective photon flux is strongly peaked at very low values of $Q^{2}$. This yields a rate sufficient to get an estimate of the transversity GPDs in the region of small $\xi(\sim 0.01)$. In the lower energy domain, which will be studied in details at JLab, and with the same cuts as above, estimates of the cross sections are: 


$$
\sigma_{\gamma N \rightarrow \pi^{+} \rho_{T}^{0} N^{\prime}}\left(S_{\gamma N}=10 \mathrm{GeV}^{2}\right) \simeq 15 \mathrm{nb} \quad \sigma_{\gamma N \rightarrow \pi^{+} \rho_{T}^{0} N^{\prime}}\left(S_{\gamma N}=20 \mathrm{GeV}^{2}\right) \simeq 33 \mathrm{nb} .
$$

The high electron beam luminosity of JLab@12GeV should thus allow for a detailed analysis.

In conclusion, the process discussed here is promissing in order to get access to the chiralodd transversity GPDs, both at low real or almost real photon energies within the JLab@12GeV upgraded facility, both in Hall B and Hall D, and at higher photon energies with the COMPASS experiment. We note that our proposal is built on known leading twist factorization theorems, contrarily to other attempts to access transversity GPDs [16]. At large center of mass energy, for chiral-even $t$-channel exchange, hard exclusive vector meson production beyond leading twist has been consistently described in Ref. [17]. This might be extended for chiral-odd quantities.

Work supported by the grants ANR-06-JCJC-0084, Polish N202 249235, DFG (KI-623/4).

\section{References}

[1] J. P. Ralston and D. E. Soper, Nucl. Phys. B 152, 109 (1979); X. Artru and M. Mekhfi, Z. Phys. C 45, 669 (1990); J. L. Cortes et al., Z. Phys. C 55, 409 (1992); R. L. Jaffe and X. D. Ji, Phys. Rev. Lett. 67, $552(1991)$.

[2] V. Barone, A. Drago and P. G. Ratcliffe, Phys. Rept. 359, 1 (2002); M. Anselmino, arXiv:hep-ph/0512140; B. Pire and L. Szymanowski, Phys. Rev. Lett. 103, 072002 (2009).

[3] M. Diehl, Eur. Phys. J. C 19, 485 (2001).

[4] M. Diehl et al., Phys. Rev. D 59, 034023 (1999); J. C. Collins and M. Diehl, Phys. Rev. D 61, 114015 (2000).

[5] D. Yu. Ivanov et al., Phys. Lett. B 550, 65 (2002); R. Enberg et al., Eur. Phys. J. C 47, 87 (2006).

[6] S. Kumano et al., Phys. Rev. D 80, 074003 (2009).

[7] M. E. Beiyad et al., arXiv:0911.2611 [hep-ph]; Phys. Lett. B 688, 154 (2010); PoS DIS2010:252,2010 [arXiv:1006.0740 [hep-ph]]; arXiv:1007.2710 [hep-ph]; arXiv:1011.0840 [hep-ph].

[8] S. Scopetta, Phys. Rev. D 72, 117502 (2005).

[9] M. Pincetti et al., Phys. Rev. D 72, 094029 (2005) and Czech. J. Phys. 56, F229 (2006).

[10] M. Wakamatsu, Phys. Rev. D 79, 014033 (2009); D. Chakrabarti et al., Phys. Rev. D 79, 034006 (2009).

[11] M. Gockeler et al., Phys. Rev. Lett. 98, 222001 (2007) and Phys. Lett. B 627, 113 (2005).

[12] G. P. Lepage and S. J. Brodsky, Phys. Rev. D 22, 2157 (1980).

[13] G. R. Farrar et al., Phys. Rev. Lett. 62, 2229 (1989).

[14] M. Anselmino et al., Phys. Rev. D 75 (2007) 054032.

[15] M. Burkardt, Phys. Rev. D 62 (2000) 071503; J. P. Ralston and B. Pire, Phys. Rev. D 66 (2002) 111501; M. Burkardt, Phys. Rev. D 72, 094020 (2005); M. Diehl and Ph. Hagler, Eur. Phys. J. C 44, 87 (2005); A. Mukherjee, D. Chakrabarti and R. Manohar, AIP Conf. Proc. 1149, 533 (2009).

[16] S. Ahmad, G. R. Goldstein and S. Liuti, Phys. Rev. D 79, 054014 (2009); S. V. Goloskokov and P. Kroll, Eur. Phys. J. C 65, 137 (2010).

[17] I. V. Anikin et al., AIP Conf. Proc. 1105, 390 (2009); Phys. Lett. B 682, 413 (2010); Acta Phys. Polon. B 40, 2131 (2009); PoS EPS-HEP2009:070,2009 [arXiv:0909.4038 [hep-ph]]; arXiv:0909.4042 [hep-ph]; Nucl. Phys. B 828, 1 (2010); arXiv:1006.1477 [hep-ph]; arXiv:1011.1854 [hep-ph]. 